\title{
Defunctioning ileostomy and mechanical bowel preparation may contribute to development of low anterior resection syndrome
}

\author{
Michat M. Nowakowski ${ }^{1}$, Mateusz Rubinkiewicz ${ }^{2}$, Natalia Gajewska², Grzegorz Torbicz ${ }^{2}$, Michał Wysocki $^{2,3}$, \\ Piotr Małczak ${ }^{2,3}$, Piotr Major ${ }^{2,3}$, Mateusz Wierdak ${ }^{2,3}$, Andrzej Budzyński ${ }^{2,3}$, Michat Pędziwiatr ${ }^{2,3}$ \\ ${ }^{1}$ Department of Medical Education, Jagiellonian University Medical College, Krakow, Poland \\ $2^{2} 2^{\text {nd }}$ Department of General Surgery, Jagiellonian University Medical College, Krakow, Poland \\ ${ }^{3}$ Centre for Research, Training and Innovation in Surgery (CERTAIN Surgery), Krakow, Poland
}

Videosurgery Miniinv 2018; 13 (3): 306-314 DOI: https://doi.org/10.5114/wiitm.2018.76913

\begin{abstract}
Introduction: Laparoscopic surgery is an approved technique in colorectal cancer treatment. Functional and quality-of-life studies have revealed significant changes in faecal continence.

Aim: To assess the incidence and risk factors of low anterior resection syndrome (LARS) in patients undergoing rectal resections for cancer.

Material and methods: We enrolled patients undergoing rectal resections in a general surgery department of a university hospital. The primary outcomes were the Jorge-Wexner scale and the LARS score 6 months after the end of treatment. The secondary outcomes were the risk factors for LARS development.

Results: Fifty-six patients were included; 15 (26\%) developed major LARS and 10 (18\%) had minor LARS at 6 months. In univariate analysis the risk factors were: preoperative radiotherapy ( $p<0.001, O R=11.9,95 \% \mathrm{Cl}: 2.98-47.48)$; shorter distance of the tumour from the anal verge ( $p=0.001, O R=0.69,95 \% \mathrm{Cl}: 0.55-0.86)$; bowel preparation $(p=0.01, O R=6.27,95 \% \mathrm{Cl}: 1.51-26.07)$; low anterior rectal resection ( $p=0.01, O R=17.07,95 \% \mathrm{Cl}: 1.86-156.83)$; and protective ileostomy ( $p=0.001, O R=15.97,95 \% \mathrm{Cl}: 4.07-61.92)$. The risk factors for a higher Jorge-Wexner score in univariate analysis were greater diameter of tumour $(p=0.035)$, radiotherapy $(p=0.001)$, shorter distance from the anal verge $(p=0.002)$, bowel preparation $(p=0.042)$, low anterior rectal $(L A R)(p=0.01)$, ileostomy $(p=0.001)$, perioperative complications $(p=0.032)$, and readmission within 30 days $(p=0.034)$. In the multivariate analysis, readmissions and perioperative complications were significant.

Conclusions: In addition to typically described risk factors, two new ones have been identified. Mechanical bowel preparation and defunctioning ileostomy may also contribute to LARS development. However, due to the limitations of this study our observations require further confirmation in future trials.
\end{abstract}

Key words: risk factors, rectal cancer, low anterior resection, low anterior resection syndrome, mechanical bowel preparation, ileostomy.

\section{Introduction}

Laparoscopic surgery is an approved technique in colorectal cancer treatment. Lower rates of perioperative complications and at least equal oncological results have been proven in randomised controlled tri- als and confirmed with meta-analyses [1, 2]. Despite technical improvements to abdominoperineal resection [3], over the last two decades there has been a significant shift in types of resections performed for rectal cancer treatment [4]. Improved surgical techniques and technology, introduction of neoadju- 
vant treatment, and better understanding of disease biology have increased the proportions of patients undergoing sphincter-preserving procedures and decreased the percentage of abdominoperineal amputations [5]. At the same time, functional and quality-of-life assessment studies have brought to light significant changes in those domains resulting from the applied treatments [6]. Symptoms include, but are not limited to, difficulties with flatus and stool control, urgency to defecate, loss of sensory functions, emptying problems, and many others [7]. Prevalence of those symptoms amongst low anterior resection patients differs between the studies depending on definitions of low anterior resection syndrome (LARS) or its components and sensitivity of applied measurement methodologies, but anything between 30\% and $90 \%$ of patients may be involved. Different symptoms of low LARS have different effects on patients' wellbeing. One of the most debilitating components of LARS is faecal incontinence. This influences many aspects of patient functioning, which is reflected in quality-of-life changes, mainly in coping and embarrassment domains [8]. Some speculation has been made on the exact aetiology of LARS. Disruption of somatic and/or autonomic innervations [9-11] and changes in rectal reservoir functions due to alterations of rectal compliance or rectal ampulla volume have been suggested as possible causes [9, 12]. In addition, changes related to neoadjuvant treatment, probably mostly those attributed to radiotherapy, influence the functional results [13-15]. Loss of sensory function of the recto-anal transitory zone is also thought to influence discriminatory function and enhance the probability of faecal incontinence [16].

There is little difference between open and laparoscopic approaches in terms of rectal reservoir function, since this is dependent on anastomotic techniques and perhaps on the length of proximal resection, but there might be a difference in terms of preservation of neural structures. Key logic behind this speculation would be better visualisation and precision of dissection resulting from operating field exposure and magnification. Conversely, due to lower degrees of freedom, lack of tactile assessment and increased technical difficulty, it may be the case that laparoscopy increases the probability of LARS [17]. To date most data have referred to patients operated on with an open approach or mixed groups were analyzed. Moreover, perioperative protocols are leading to a change of traditional risk factors for postoperative complications [18]. Whether they bring benefits beyond hospital stay is still under debate.

\section{Aim}

In this study we decided to establish risk factors for LARS in patients undergoing laparoscopic total mesorectal excision (TME) for rectal cancer.

\section{Material and methods}

\section{Setting and design}

Between 2014 and 2016, prospective data collection was performed in patients undergoing transabdominal resection for the treatment of rectal cancer. All procedures were performed in a tertiary-level university teaching hospital. The annual volume of colorectal procedures exceeds 120 , and approximately 50 of those are performed for rectal cancer. Patients with histologically confirmed low and mid $(<10 \mathrm{~cm}$ from the anal verge) rectal adenocarcinoma were included in the study and were submitted to neoadjuvant chemoradiotherapy when indicated. Each patient underwent evaluation of faecal continence twice (before any treatment and 6 months after the end of treatment) or three times when undergoing neoadjuvant radiotherapy. We used the Jorge-Wexner scale and LARS score for patient assessment: major LARS was defined as a LARS score > 30; minor LARS was defined as a LARS score of 21-29 [19, 20]. Patients with any sort of faecal diversion at the time of the last evaluation (end colostomy, loop ileostomy) or those lost to follow-up were excluded from the study. All procedures were performed by the same surgical team, with the number of cases and conversion rates suggesting high placement on a learning curve [21]. Assessment of complications was performed using the Clavien-Dindo Classification, and we considered a Clavien-Dindo score of III-V to be a severe complication [22]. Each patient was treated with the standardized Enhanced Recovery after Surgery protocol with median compliance above $80 \%[23,24]$.

\section{Measured outcomes}

The primary outcome was the LARS score and Jorge-Wexner score at 6 months after surgical treatment $[13,20]$. The secondary outcomes were the risk factors for LARS development. 


\section{Operative technique}

The surgical technique has been described elsewhere as nerve preserving total mesorectal excision [25]. The anastomosis was performed using a circumferential stapler. Protective ileostomy was created for all anastomoses in the ultra-low anterior resection group and selectively in the low anterior resection group.

\section{Statistical analysis}

All data were analysed with Statistica version 13.0 PL (StatSoft Inc., Tulsa, OK, USA). The results are presented as mean, standard deviation (SD), median, and interquartile range (IQR). The study of categorical variables used the $\chi^{2}$ test of independence. The Shapiro-Wilk test was used to check for normal distribution of data, and Student's $t$ test was used for normally distributed quantitative data. For non-normally distributed quantitative variables, the Mann-Whitney $U$ test was used. A $p$-value $<0.05$ was considered statistically significant. All considerable patient- and treatment-related factors were analysed in univariate logistic regression models, then significant factors were analysed in the multiple logistic regression model in search of independent risk factors for LARS.

The study was approved by the local ethics committee. Each patient signed an informed consent form before inclusion in the study.

\section{Results}

Eighty-three patients were eligible for the study. The group design is presented in Figure 1. Based on exclusion criteria, 56 patients were included in the study. Group characteristics and perioperative outcomes are shown in Table I.

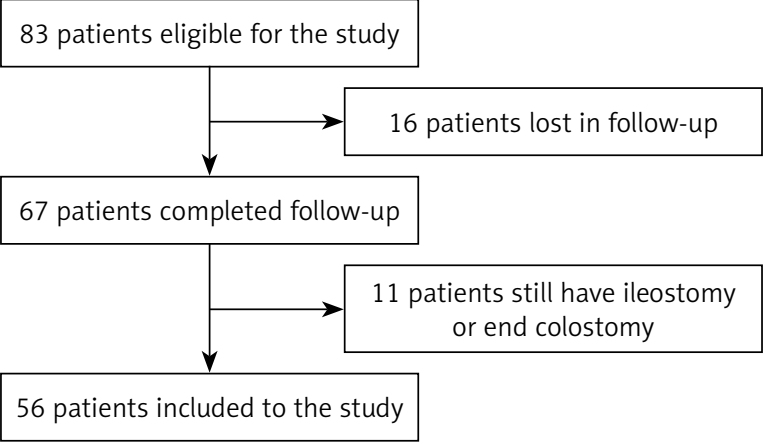

Figure 1. Study group design
Fifteen (26\%) patients developed major LARS and $10(18 \%)$ patients had symptoms of minor LARS 6 months after surgery. The risk factors of LARS in univariate analysis were as follows: preoperative radiotherapy $(\mathrm{OR}=11.9,95 \% \mathrm{Cl}: 2.98-47.48$, $p<0.001)$; bowel preparation $(\mathrm{OR}=6.27,95 \% \mathrm{Cl}$ : 1.51-26.07, $p=0.01)$; ultra-low anterior rectal resection $(\mathrm{OR}=17.07,95 \% \mathrm{Cl}: 1.86-156.83, p=0.01)$; and protective ileostomy creation $(\mathrm{OR}=15.97$, 95\% Cl: 4.07-61.92, $p=0.001)$. Greater distance of the tumour from the anal verge reduced the incidence of LARS (OR $=0.69,95 \% \mathrm{Cl}: 0.55-0.86$, $p=0.001$ ). When multivariate analysis was performed, none of the analyzed factors turned out to be a major factor contributing to LARS development. A summary of our results is included in Tables II and III.

The median Jorge-Wexner score after surgery was 3 points (IQR: $0-8$, range: $0-18)$. The risk factors for a higher Jorge-Wexner score in univariate analysis were a higher $\mathrm{T}$ parameter in the TNM classification ( $p=0.035)$, radiotherapy $(p=0.001)$, shorter distance of the tumour from the anal verge $(p=0.002)$, bowel preparation $(p=0.042)$, low anterior rectal resection $(p=0.01)$, ileostomy creation $(p=0.001)$, perioperative complications $(p=0.032)$ and readmission to the hospital within 30 days $(p=0.034)$. In multivariate analysis, readmissions and perioperative complications were a risk factors of a higher Jorge-Wexner score $(p=0.021$ and $p=0.04$, respectively).

Eighteen patients suffered from postoperative complications, including five considered severe (ClavienDindo III-V): three presented anastomotic leakage and two postoperative ileus. There were four 30-day readmissions due to late postoperative complications, none of which required surgical intervention. There were no mortalities within 30 days of the surgical procedure.

\section{Discussion}

In our study we demonstrated that a laparoscopic approach is a safe and valuable tool for rectal resections in treatment of rectal cancer. However, $44 \%$ of patients developed minor or major LARS, which remains a high incidence rate.

Protective ileostomy was found to be a risk factor for LARS development after rectal resection, confirmed both in the LARS score and the Jorge-Wexner scale. This fact is rarely reported in the literature. Similar findings were presented by Jimenez-Gomez 
Table I. General characteristics and perioperative outcomes

\begin{tabular}{|c|c|c|}
\hline \multicolumn{2}{|c|}{ Parameter } & Results \\
\hline \multicolumn{2}{|c|}{ Males/females } & $28 / 28(50 \% / 50 \%)$ \\
\hline \multicolumn{2}{|c|}{ Age, mean $\pm S D$, min.-max. [years] } & $61 \pm 11,36-83$ \\
\hline \multicolumn{2}{|c|}{ BMI, median (IQR); min.-max. [kg/m²] } & $\begin{array}{c}26.58(24.04-29.71) \\
19.00-43.51\end{array}$ \\
\hline \multicolumn{2}{|c|}{ ASA 1, n (\%) } & $2(3.57)$ \\
\hline \multicolumn{2}{|c|}{ ASA 2, n (\%) } & $50(89.29)$ \\
\hline \multicolumn{2}{|c|}{ ASA 3, n (\%) } & $4(7.14)$ \\
\hline \multicolumn{2}{|c|}{ Any significant comorbidity, $n(\%)$} & $32(57.14)$ \\
\hline \multicolumn{2}{|c|}{ Diabetes mellitus, $n(\%)$} & $11(19.64)$ \\
\hline \multicolumn{2}{|c|}{ Arterial hypertension, $n$ (\%) } & $22(39.29)$ \\
\hline \multicolumn{2}{|c|}{ Coronary artery disease, $n(\%)$} & 15 (26.79) \\
\hline \multicolumn{2}{|c|}{ Renal comorbidity, $n$ (\%) } & $2(3.57)$ \\
\hline \multicolumn{2}{|c|}{ Hepatic comorbidity, $n(\%)$} & $1(1.79)$ \\
\hline \multicolumn{2}{|c|}{ Pulmonary comorbidity, $n$ (\%) } & $1(1.79)$ \\
\hline \multirow[t]{5}{*}{$\mathrm{T}$} & 0 & $3(6 \%)$ \\
\hline & 1 & $2(4 \%)$ \\
\hline & 2 & $10(20 \%)$ \\
\hline & 3 & $31(62 \%)$ \\
\hline & 4 & $4(8 \%)$ \\
\hline \multirow[t]{3}{*}{ N } & 0 & $34(70.83 \%)$ \\
\hline & 1 & $6(12.5 \%)$ \\
\hline & 2 & $8(16.67 \%)$ \\
\hline \multirow[t]{4}{*}{ AJCC } & 0 & $2(3.57 \%)$ \\
\hline & 1 & $19(33.93 \%)$ \\
\hline & 2 & $20(35.71 \%)$ \\
\hline & 3 & $15(26.79 \%)$ \\
\hline
\end{tabular}

\begin{tabular}{|c|c|c|}
\hline \multicolumn{2}{|c|}{ Parameter } & Results \\
\hline \multicolumn{2}{|c|}{ Radiotherapy } & $23(46.94 \%)$ \\
\hline \multicolumn{2}{|c|}{ Depth, median (IQR) [cm] } & $10(7-12)$ \\
\hline \multirow[t]{3}{*}{ Depth } & 1 & $24(42.86 \%)$ \\
\hline & 2 & $23(41.07 \%)$ \\
\hline & 3 & $9(16.07 \%)$ \\
\hline \multicolumn{2}{|c|}{ Bowel preparation } & $37(66.07 \%)$ \\
\hline \multicolumn{2}{|c|}{ Pre-op. drink } & $48(85.71 \%)$ \\
\hline \multicolumn{2}{|c|}{ Locoregional anaesthesia } & $27(58.70 \%)$ \\
\hline \multicolumn{2}{|c|}{ Laparoscopic low anterior resection } & $47(83.93 \%)$ \\
\hline \multicolumn{2}{|c|}{$\begin{array}{l}\text { Laparoscopic ultra-low anterior } \\
\text { resection }\end{array}$} & $9(16.07 \%)$ \\
\hline \multicolumn{2}{|c|}{ Ileostomy } & $22(39.29 \%)$ \\
\hline \multicolumn{2}{|c|}{ Operative time, median (IQR) [min] } & $195(140-240)$ \\
\hline \multicolumn{2}{|c|}{ Blood loss, median (IQR) [ml] } & $70(50-150)$ \\
\hline \multicolumn{2}{|c|}{ Perioperative complications } & $18(32.14 \%)$ \\
\hline \multirow{3}{*}{$\begin{array}{l}\text { Clavien- } \\
\text { Dindo } \\
\text { classifi- } \\
\text { cation }\end{array}$} & 1 & $11(19.64 \%)$ \\
\hline & $\|$ & $2(3.57 \%)$ \\
\hline & III & $5(8.93 \%)$ \\
\hline \multicolumn{2}{|c|}{ LOS, median (IQR) [days] } & $5(3-7)$ \\
\hline \multicolumn{2}{|c|}{ Readmissions } & $4(8.33 \%)$ \\
\hline \multicolumn{2}{|c|}{ Postoperative Wexner, median (IQR) } & $3(0.5-8)$ \\
\hline \multicolumn{2}{|c|}{ Minor LARS } & $8(14.29 \%)$ \\
\hline \multicolumn{2}{|c|}{ Major LARS } & $15(26.79 \%)$ \\
\hline
\end{tabular}

et al. [26]. Moreover, data from the LAARIS Trial Management Group indicate that a longer time from the initial procedure to ileostomy reversal increases LARS incidence [27]. That finding was not confirmed in the above-mentioned study by Jimènez-Rodriguez et al. [28]. Ileostomy creation decreases the rate of anastomotic leakage after rectal resections, although it may also increase the overall complication rate [29]. Therefore, the decision of ileostomy creation is even more challenging. Seeing that the literature data is inconsistent, further research in this field is needed. To date it seems that if ileostomy is performed it should be reversed within 6 months, since the LARS incidence in that case may be lower [27]. Moreover, there is evidence that very early ileostomy closure is beneficial and does not influence adjuvant treatment [30-32].

A novel finding in our study is that mechanical bowel preparation (MBP) is associated with increased risk of LARS. Although it was said that patients do not benefit from bowel preparation [33, 34], recently this opinion has been challenged again. Some new studies have shown that MBP combined with antibiotic preparation efficiently reduces perioperative complications in colorectal surgery [35-38]. These studies are, however, prone to selection bias - patients in the no-MBP group were characterized by a higher comorbidity rate, more advanced stage of cancer, higher rate of renal and liver failure as well as more frequent steroid use. In addition, the most recent analysis based on the same registry data as previous analyses with propensity score matching confirmed that it is oral antibiotics rather than MBP 
Table II. Factors influencing odds ratio for LARS

\begin{tabular}{|c|c|c|c|}
\hline Parameter & OR & $95 \% \mathrm{Cl}$ & $P$-value \\
\hline \multicolumn{4}{|l|}{ Univariate: } \\
\hline Males vs. females & 2.11 & $0.70-6.41$ & 0.177 \\
\hline Age & 0.98 & $0.93-1.03$ & 0.364 \\
\hline BMI & 0.95 & $0.84-1.08$ & 0.448 \\
\hline ASA & 0.55 & $0.09-3.22$ & 0.499 \\
\hline Comorbidity & 0.71 & $0.24-2.13$ & 0.531 \\
\hline Diabetes mellitus & 0.25 & $0.05-1.36$ & 0.102 \\
\hline Arterial hypertension & 1.35 & $0.44-4.09$ & 0.592 \\
\hline Coronary artery disease & 0.42 & $0.11-1.59$ & 0.192 \\
\hline Renal comorbidity & $\mathrm{n} / \mathrm{a}$ & & \\
\hline Hepatic comorbidity & 2.07 & $0.001-9.99$ & 0.999 \\
\hline Pulmonary comorbidity & $\mathrm{n} / \mathrm{a}$ & & \\
\hline T & 0.60 & $0.30-1.18$ & 0.131 \\
\hline $\mathrm{N}$ & 0.91 & $0.42-1.98$ & 0.812 \\
\hline AJCC & 0.93 & $0.49-1.76$ & 0.820 \\
\hline Radiotherapy & 11.9 & $2.98-47.48$ & $<0.001$ \\
\hline Depth & 0.69 & $0.55-0.86$ & 0.001 \\
\hline Bowel preparation & 6.27 & $1.51-26.07$ & 0.010 \\
\hline Pre-op. drink & 1.19 & $0.25-5.77$ & 0.825 \\
\hline Locoregional anaesthesia & 1.48 & $0.44-5.00$ & 0.515 \\
\hline $\begin{array}{l}\text { Laparoscopic ultra-low } \\
\text { anterior resection vs. laparo- } \\
\text { scopic low-anterior resection }\end{array}$ & 17.07 & $1.86-156.83$ & 0.010 \\
\hline Vessels 2 vs. 1 & 1.12 & $0.34-3.73$ & 0.852 \\
\hline Ileostomy & 15.87 & $4.07-61.92$ & $<0.001$ \\
\hline Operative time & 0.99 & $0.98-1.01$ & 0.671 \\
\hline Blood loss & 0.99 & $0.98-1.00$ & 0.127 \\
\hline Perioperative complications & 1.71 & $0.54-5.47$ & 0.352 \\
\hline Clavien-Dindo & 2.33 & $0.34-15.83$ & 0.378 \\
\hline LOS & 1.12 & $0.98-1.28$ & 0.076 \\
\hline Readmissions & 4.33 & $0.39-48.00$ & 0.220 \\
\hline \multicolumn{4}{|l|}{ Multivariate: } \\
\hline Radiotherapy & 2.60 & $0.28-24.05$ & 0.388 \\
\hline Depth & 1.89 & $0.51-7.03$ & 0.330 \\
\hline Bowel preparation & 2.40 & $0.28-20.84$ & 0.413 \\
\hline $\begin{array}{l}\text { Laparoscopic ultra-low anterior } \\
\text { resection vs. laparoscopic low } \\
\text { anterior resection }\end{array}$ & 3.27 & $0.27-39.69$ & 0.339 \\
\hline Ileostomy & 1.80 & $0.14-23.80$ & 0.648 \\
\hline
\end{tabular}


Table III. Regression models of parameters influencing postoperative Wexner score

\begin{tabular}{|c|c|c|}
\hline Parameter & Parameter \pm SD & $P$-value \\
\hline \multicolumn{3}{|l|}{ Univariate: } \\
\hline Males vs. females & $-0.43 \pm 0.82$ & 0.602 \\
\hline Age & $-0.001 \pm 0.074$ & 0.986 \\
\hline BMI & $-0.343 \pm 0.171$ & 0.050 \\
\hline ASA & $-1.048 \pm 2.514$ & 0.678 \\
\hline Comorbidity & $-0.198 \pm 0.827$ & 0.812 \\
\hline Diabetes mellitus & $-1.170 \pm 1.019$ & 0.256 \\
\hline Arterial hypertension & $0.398 \pm 0.837$ & 0.636 \\
\hline Coronary artery disease & $-1.189 \pm 0.911$ & 0.197 \\
\hline Renal comorbidity & $-0.833 \pm 2.204$ & 0.707 \\
\hline Hepatic comorbidity & $-1.836 \pm 3.083$ & 0.554 \\
\hline Pulmonary comorbidity & $-0.309 \pm 3.093$ & 0.921 \\
\hline T & $1.747 \pm 0.803$ & 0.035 \\
\hline $\mathrm{N}$ & $-0.060 \pm 1.040$ & 0.954 \\
\hline $\mathrm{AJCC}$ & $-0.199 \pm 0.959$ & 0.836 \\
\hline Radiotherapy & $3.019 \pm 0.816$ & 0.001 \\
\hline Depth & $-0.823 \pm 0.203$ & 0.002 \\
\hline Bowel preparation & $1.734 \pm 0.832$ & 0.042 \\
\hline Pre-op. drink & $-0.448 \pm 1.169$ & 0.703 \\
\hline Locoregional anaesthesia & $-0.640 \pm 0.897$ & 0.479 \\
\hline $\begin{array}{l}\text { Laparoscopic ultra-low anterior resection } \\
\text { vs. laparoscopic low-anterior resection }\end{array}$ & $2.816 \pm 1.047$ & 0.010 \\
\hline Vessels 2 vs. 1 & $0.962 \pm 0.908$ & 0.295 \\
\hline Ileostomy & $2.757 \pm 0.750$ & 0.001 \\
\hline Operative time & $0.009 \pm 0.013$ & 0.486 \\
\hline Blood loss & $-0.008 \pm 0.006$ & 0.233 \\
\hline Perioperative complications & $1.845 \pm 0.840$ & 0.032 \\
\hline 3 vs. 2-1 Clavien-Dindo & $1.204 \pm 1.427$ & 0.403 \\
\hline Clavien-Dindo & $1.289 \pm 0.868$ & 0.143 \\
\hline LOS & $0.211 \pm 0.163$ & 0.201 \\
\hline Readmissions & $3.523 \pm 1.615$ & 0.034 \\
\hline \multicolumn{3}{|l|}{ Multivariate: } \\
\hline Constant term & $10.105 \pm 5.933$ & 0.099 \\
\hline $\mathrm{T}$ & $-1.212 \pm 0.942$ & 0.208 \\
\hline Radiotherapy & $4.459 \pm 2.878$ & 0.132 \\
\hline Depth & $-0.393 \pm 0.335$ & 0.250 \\
\hline $\begin{array}{l}\text { Laparoscopic ultra-low anterior resection } \\
\text { vs. laparoscopic low-anterior resection }\end{array}$ & $1.379 \pm 2.346$ & 0.561 \\
\hline Ileostomy & $-2.087 \pm 3.402$ & 0.544 \\
\hline Perioperative complications & $3.578 \pm 1.669$ & 0.040 \\
\hline Readmissions & $6.696 \pm 2.902$ & 0.028 \\
\hline
\end{tabular}


that reduced complication rates [39]. Therefore if MBP seems to be ineffective and also may contribute to LARS development the decision to perform MBP should be carefully considered and well justified. Mechanical bowel preparation may be necessary when defunctioning ileostomy or transanal TME is planned; nonetheless van't Sant et al. questioned that hypothesis in a large multicenter study [40]. Also Pittet et al. suggested that rectal enema may successfully replace MBP [41]. Bearing in mind our results the decision of MBP is still uncertain and requires extensive investigation.

Perioperative radiotherapy is a risk factor of faecal incontinence 6 months after the surgery. Other authors also identified neoadjuvant radiotherapy as a factor leading to LARS $[26,42,43]$. Postoperative radiotherapy also causes deterioration of anal sphincter function [44, 45]. The effect of radiotherapy is not only clinically significant, but also is visible in manometric studies [46]. Nevertheless, radiotherapy significantly improves overall survival and disease-free survival, and thus the elimination of this part of modern treatment is not possible [47, 48]. However, more cautious selection of patients for preoperative radiotherapy should be performed in order to limit anal sphincter dysfunction.

A shorter distance from the anal verge and a larger size of the tumour are associated with LARS in the postoperative period. This is probably caused by the necessity of more extensive surgical techniques in order to gain proper resection margins. The type of the procedure (partial mesorectal excision vs. total mesorectal excision) is also considered as a risk factor of LARS [49].

Laparoscopic technique resulted in a lower LARS score according to Nuytens et al. [42]. In contrast, a meta-analysis performed by Lim et al. showed no difference between minimally invasive and open approaches regarding sexual and bladder functions [50]. There are few published studies comparing laparoscopic and open approaches in assessing long-term functional outcomes regarding anal sphincters. We had no open cases or conversions in our material, so such an analysis was impossible. Nevertheless the incidence of LARS in our material is comparable to other authors who use an open approach [49]. This suggests no superiority of minimally invasive techniques, but further investigations including RCTs are necessary.

Anastomosis with mechanical staplers reduces the incidence of LARS [43]. In our study, all anasto- moses were performed using a mechanical stapler, so we cannot draw any conclusions regarding that variable.

Our study has some limitations, amongst which the relatively small number of included patients may result in a type II error. Moreover, we did not perform an analysis between LARS and time to ileostomy closure. Lastly, although some risk factors have been identified, they were not confirmed in the multivariate model, which is likely due to the limited number of cases or number of influencing variables leading to study group heterogeneity. Therefore, larger trials are needed in order to confirm our findings.

\section{Conclusions}

In this study we were able to confirm that traditional risk factors for LARS are also present in laparoscopic rectal surgery. In addition, two new potential risk factors have been identified. Mechanical bowel preparation, being a part of perioperative care in rectal surgery, has been suggested to contribute to LARS development. In addition, defunctioning ileostomy may also have an impact on impaired function of the neorectum in the early postoperative period. However, due to the limitations of this study our observations require further confirmation in future trials.

\section{Conflict of interest}

The authors declare no conflict of interest.

\section{References}

1. Di B, Li Y, Wei K, et al. Laparoscopic versus open surgery for colon cancer: a meta-analysis of 5-year follow-up outcomes. Surg Oncol 2013; 22: e39-43.

2. Małczak P, Mizera M, Witowski J, et al. Is the laparoscopic approach for rectal cancer superior to open surgery? A systematic review and meta-analysis on short-term surgical outcomes. Videosurgery Miniinv 2018; 13: 129-40.

3. Yamamoto T, Kanazawa A, Matsubara H, et al. Safety and usefulness of needle-guided resection of levator muscles in laparoscopic abdominoperineal resection for low rectal cancer. Videosurgery Miniinv 2016; 11: 186-91.

4. Mohammed S, Anaya DA, Awad SS, et al. Sphincter preservation rates after radical resection for rectal cancer in the United States veteran population: opportunity for improvement in early disease. Ann Surg Oncol 2015; 22: 216-23.

5. Jorgensen ML, Young JM, Dobbins TA, et al. Assessment of abdominoperineal resection rate as a surrogate marker of hospital quality in rectal cancer surgery. Br J Surg 2013; 100: 1655-63.

6. Bryant CLC, Lunniss PJ, Knowles $\mathrm{CH}$, et al. Anterior resection syndrome. Lancet Oncol 2012; 13: e403-8. 
7. Juul T, Ahlberg M, Biondo S, et al. Low anterior resection syndrome and quality of life. Dis Colon Rectum 2014; 57: 585-91.

8. Rockwood TH. Impact of fecal incontinence on quality of life. In: Fecal Incontinence: Diagnosis and Treatment. Springer-Verlag, Italy 2007; 73-7.

9. Reibetanz J, Kim M, Germer C, et al. Late complications and functional disorders after rectal resection: prevention, detec tion and therapy. Chirurg 2015; 86: 326-31.

10. Sałówka J, Nowakowski M, Wałęga P, et al. Influence of extent of rectal resection on superficial electromyography of the ex ternal anal sphincter in patients with rectal cancer. Proktologia 2008; 8: 237-53.

11. Nowakowski M, Tomaszewski K, Herman RM, et al. Developing a new electromyography-based algorithm to diagnose the etiology of fecal incontinence. Int J Colorectal Dis 2014; 29: 747-54.

12. Rubin F, Douard R, Wind P. The functional outcomes of coloanal and low colorectal anastomoses with reservoirs after low rectal cancer resections. Am Surg 2014; 80: 1222-9.

13. Bregendahl S, Emmertsen KJ, Lous J, et al. Bowel dysfunction after low anterior resection with and without neoadjuvant therapy for rectal cancer: a population-based cross-sectional study. Color Dis 2013; 15: 1130-9.

14. Lundby L, Krogh K, Jensen VJ, et al. Long-term anorectal dysfunction after postoperative radiotherapy for rectal cancer. Dis Colon Rectum 2005; 48: 1343-9.

15. Yeoh E, Sun WM, Russo A, et al. A retrospective study of the effects of pelvic irradiation for gynecological cancer on anorectal function. Int J Radiat Oncol Biol Phys 1996; 35: 1003-10.

16. Ziv Y, Zbar A, Bar-Shavit Y, et al. Low anterior resection syndrome (LARS): cause and effect and reconstructive considerations. Tech Coloproctol 2013; 17: 151-62.

17. Chew MH, Yeh YT, Lim E, et al. Pelvic autonomic nerve preservation in radical rectal cancer surgery: changes in the past 3 decades. Gastroenterol Rep 2016; 4: 173-85.

18. Pędziwiatr M, Pisarska M, Kisielewski M, et al. Is ERAS in laparoscopic surgery for colorectal cancer changing risk factors for delayed recovery? Med Oncol 2016; 33: 25.

19. Emmertsen KJ, Laurberg S, Emmertsen KJ, Laurberg S. Low anterior resection syndrome score. Ann Surg 2012; 255: 922-8.

20. Jorge JM, Wexner SD. Etiology and management of fecal incontinence. Dis Colon Rectum 1993; 36: 77-97.

21. Luglio G, De Palma GD, Tarquini R, et al. Laparoscopic colorectal surgery in learning curve: role of implementation of a standardized technique and recovery protocol. A cohort study. Ann Med Surg 2015; 4: 89-94.

22. Clavien PA, Barkun J, De Oliveira ML, et al. The clavien-dindo classification of surgical complications: five-year experience. Ann Surg 2009; 250: 187-96.

23. Pisarska M, Pędziwiatr M, Małczak P, et al. Do we really need the full compliance with ERAS protocol in laparoscopic colorectal surgery? A prospective cohort study. Int I Surg 2016; 36: 377-82.

24. Pędziwiatr M, Pisarska M, Kisielewski M, et al. ERAS protocol in laparoscopic surgery for colonic versus rectal carcinoma: are there differences in short-term outcomes? Med Oncol 2016; 33: 56.
25. Gordon PH, Nivatvongs S. Principles and Practice of Surgery for the Colon, Rectum, and Anus. $3^{\text {th }}$ ed. CRC Press; 2007; 1360.

26. Jimenez-Gomez LM, Espin-Basany E, Trenti L, et al. Factors associated with low anterior resection syndrome after surgical treatment of rectal cancer. Color Dis 2018; 20: 195-200.

27. Hughes DL, Cornish J, Morris C, et al. Functional outcome following rectal surgery-predisposing factors for low anterior resection syndrome. Int J Colorectal Dis 2017; 32: 691-7.

28. Jiménez-Rodríguez RM, Segura-Sampedro JJ, Rivero-Belenchón I, et al. Is the interval from surgery to ileostomy closure a risk factor for low anterior resection syndrome? Colorectal Dis 2017; 19: 485-90.

29. Pisarska M, Gajewska N, Małczak P, et al. Defunctioning ileostomy reduces leakage rate in rectal cancer surgery - systematic review and meta-analysis. Oncotarget 2018; 9: 20816-25.

30. Zhou MW, Wang ZH, Chen ZY, et al. Advantages of early preventive ileostomy closure after total mesorectal excision surgery for rectal cancer: an institutional retrospective study of 123 consecutive patients. Dig Surg 2017; 34: 305-11.

31. Menahem B, Lubrano J, Vallois A, et al. Early closure of defunctioning loop ileostomy: is it beneficial for the patient? A meta-analysis. World J Surg 2018; 27: doi: 10.1007/s00268-018-4603-0.

32. Farag S, Rehman S, Sains P. Early vs delayed closure of loop defunctioning ileostomy in patients undergoing distal colorectal resections: an integrated systematic review and meta-analysis of published randomized controlled trials. Color Dis 2017; 19: 1050-7.

33. Güenaga KF, Matos D, Wille-Jørgensen P. Mechanical bowel preparation for elective colorectal surgery. Cochrane Database Syst Rev 2011; 7: CD001544.

34. Courtney DE, Kelly ME, Burke JP, et al. Postoperative outcomes following mechanical bowel preparation before proctectomy: a meta-analysis. Colorectal Dis 2015; 17: 862-9.

35. Moghadamyeghaneh Z, Hanna MH, Carmichael JC, et al. Nationwide analysis of outcomes of bowel preparation in colon surgery. I Am Coll Surg 2015; 220: 912-20.

36. Scarborough JE, Mantyh CR, Sun Z, et al. Combined mechanical and oral antibiotic bowel preparation reduces incisional surgical site infection and anastomotic leak rates after elective colorectal resection. Ann Surg 2015; 262: 331-7.

37. Kiran RP, Murray ACA, Chiuzan C, et al. Combined preoperative mechanical bowel preparation with oral antibiotics significantly reduces surgical site infection, anastomotic leak, and ileus after colorectal surgery. Ann Surg 2015; 262: 416-25.

38. Morris MS, Graham LA, Chu DI, et al. Oral antibiotic bowel preparation significantly reduces surgical site infection rates and readmission rates in elective colorectal surgery. Ann Surg 2015; 261: 1034-40.

39. Klinger AL, Green H, Monlezun DJ, et al. The role of bowel preparation in colorectal surgery: results of the 2012-2015 ACS-NSQIP Data. Ann Surg 2017; doi: 10.1097/SLA.0000000000002568.

40. van't Sant HP, Weidema WF, Hop WCJ, et al. The influence of mechanical bowel preparation in elective lower colorectal surgery. Ann Surg 2010; 251: 59-63.

41. Pittet $\mathrm{O}$, Nocito $\mathrm{A}, \mathrm{Balke} \mathrm{H}$, et al. Rectal enema is an alternative to full mechanical bowel preparation for primary rectal cancer surgery. Color Dis 2015; 17: 1007-10. 
42. Nuytens F, Develtere D, Sergeant G, et al. Perioperative radiotherapy is an independent risk factor for major LARS: a cross-sectional observational study. Int I Color Dis 2018; doi: 10.1007/s00384-018-3043-5.

43. Trenti L, Galvez A, Biondo S, et al. Quality of life and anterior resection syndrome after surgery for mid to low rectal cancer: a cross-sectional study. Eur J Surg Oncol 2018; 44: 1031-9.

44. Romaniszyn M, Richter P, Walega P, et al. Low-anterior-resection syndrome. How does neoadjuvant radiotherapy and low resection of the rectum influence the function of anal sphincters in patients with rectal cancer? Preliminary results of a functional assessment study. PolJ Surg 2012; 84: 177-83.

45. Haas S, Faaborg P, Liao D, et al. Anal sphincter dysfunction in patients treated with primary radiotherapy for anal cancer: a study with the functional lumen imaging probe. Acta Oncol (Madr) 2018; 57: 465-72.

46. Ihnát P, Slívová I, Tulinsky L, et al. Anorectal dysfunction after laparoscopic low anterior rectal resection for rectal cancer with and without radiotherapy (manometry study). I Surg Oncol 2018; 117: 710-6.

47. Colorectal Cancer Collaborative G. Adjuvant radiotherapy for rectal cancer: a systematic overview of 8,507 patients from 22 randomised trials. Lancet 2001; 358: 1291-304.

48. Kapiteijn E, Marijnen CA, Nagtegaal ID, et al. Preoperative radiotherapy combined with total mesorectal excision for resectable rectal cancer. N Engl J Med 2001; 345: 638-46.

49. Kupsch J, Jackisch T, Matzel KE, et al. Outcome of bowel function following anterior resection for rectal cancer - an analysis using the low anterior resection syndrome (LARS) score. Int J Colorectal Dis 2018; 33: 787-98.

50. Lim RS, Yang TX, Chua TC. Postoperative bladder and sexual function in patients undergoing surgery for rectal cancer: a systematic review and meta-analysis of laparoscopic versus open resection of rectal cancer. Techn Coloproctol 2014; 18 993-1002.

Received: 30.05.2018, accepted: 13.06.2018. 